\title{
Using Process Mining and Control Charts to Improve the Reliability of Road Freight Transportation
}

\author{
Aleksey Dorofeev ${ }^{1,2 *}$, Valery Kurganov ${ }^{3}$, and Mikhail Gryaznov ${ }^{4}$ \\ ${ }^{1}$ Financial University under the Government of the Russian Federation, 125993, Moscow, \\ Leningradsky Prospekt, 49, Russia \\ ${ }^{2}$ Higher School of Economics - National Research University, 119049, Moscow, ul. Shabolovka, d. \\ 28/11, Russia \\ ${ }^{3}$ Tver State University, 170100, Tver, Zhelyabova, 33, Russia \\ ${ }^{4}$ Nosov Magnitogorsk State Technical University, 455000, Russia, Chelyabinsk Region, \\ Magnitogorsk, Lenin street, 38, Russia
}

\begin{abstract}
Management of a process assumes the availability of metrics to reflect its status. During road freight transportation, nowadays various sensors provide you with a full picture of what is happening with the vehicle and freight. Accordingly, in case of deviation from the specified transportation parameters, the company's CEO can exercise his/her management to adjust the transportation process. However, the influence of the human factor on the reliability of transportation is still considerable. So, methods and approaches are required to assess actions and business activities of personnel quickly and efficiently. It is currently relevant for the transportation process not only to collect data on driver behavior, but also to deeply analyze the behavior of operators of the fleet management information system.
\end{abstract}

\section{Introduction}

Despite numerous successful examples of the digitalization development in various economy industries, a person is still the main part of production chains, and the successful business results often rely on his/her actions $[1,2]$. The influence of the human factor in the services sector is still dominant, and the efficiency of a service company as a whole critically depends on well-coordinated, motivated work of employees, and partner relations with each other, as well as attitude to their company. In turn, the organizational structure of the company, the attitude of management to employees, the schedule and workplace conditions, the nature of operations leave a serious imprint on the personnel functioning [3]. Today, many studies, methods and approaches are available devoted to analyzing the company business, optimizing the personnel work, improving the efficiency of business processes, which originate from F. Taylor's early 20th-century papers on the scientific organization of labor $[4,5,6]$. However, these days, due to digitalization, it is relevant to

\footnotetext{
* Corresponding author: andorofeev@fa.ru
} 
study the organization and optimization of personnel work in the context of humancomputer interaction. Earlier, when the introduction of IT was local and was mainly a prerequisite for improving existing business processes, the influence of the human factor in the human-computer system in the service sector was not critical. In today's the digital transformation era, when IT is, in fact, the nervous system of any company, mistakes or malicious actions by operators can result in disruption of the sustainable operation of the company.

\section{Digital Control Means for Managing the Reliability of Freight Transportation}

\subsection{Factors Affecting Transportation Reliability}

Road freight transportation is another field where IT is introduced heavily. Due to GPS monitoring, the operator can receive online information about the location of the truck, and determine its deviations from the route or from the delivery schedule in real time. In modern conditions, consumers are very demanding to delivery deadlines, and deviation from the delivery time can greatly affect customer experience. Deviations from the schedule can be caused by various reasons, e.g., a vehicle malfunction, bad road or weather conditions, as well as a human factor related to the driver's work. In addition, there are other factors that characterize the deviation of the transportation process from the transportation conditions approved in the contract with the customer. These include various issues related to freight, such as damage to freight, loss of freight, as well as issues related to documents, such as incorrect or late registration [7]. Such deviations can be interpreted as failures in terms of the reliability theory. Accordingly, it is of practical interest to deliver freight with a given target reliability indicator, which is an integral value that characterizes the probability of failures associated with both the vehicle and the freight, road and weather conditions, documents and personnel work [8].

In this context, the reliability of road freight transportation in most studies is considered from the point of view of the human factor in terms of the drivers' work analysis. Clearly, safety of freight, fast and accident-free delivery in harsh weather and road conditions, as well as efficient operation of the vehicle depend on the experience, skills and motivation of drivers. Yet, there is another aspect related to the transportation process, which is determined by the human factor. This refers to the work of the management personnel who register applications for transportation, prepare documents for dispatching freight and releasing vehicles on routes, arrange repairs and maintenance of rolling stock, plan transportation activities, etc. Yet, the business activities of this employee category have been almost opaque to decision makers until recently, in contrast to the actions of the driver, which are controlled by GPS devices. Indeed, the built-in sensors of various onboard systems in modern trucks assess not only the work of the vehicle itself, but also the work of the driver. A number of truck models also include driver digital assistants, which tell them the optimal actions while driving [9]. Moreover, there are technologies that allow to assess the degree of fatigue of drivers, for example, using cameras to monitor blinking.

\subsection{Means for Analyzing the Impact of the Human Factor on the Transportation Reliability}

At the same time, much less attention is paid to monitoring the work of freight transportation dispatchers. Until recently, this has been due to the fact that for the study of their general business activities two methods were mainly used in practice: interviewing 
and monitoring. Interviewing managers is a basic method for studying the business processes of a company, which is widely used to optimize the work of a particular company. However, interviewing largely depends on the communication skills, experience and personal competencies of the interviewer, which allows him/her to win over the managers being interviewed and then correctly formalize their points of view on business activities. Obviously, the subjective factor associated with the experience and skills of the managers participating in the survey would have a significant impact in this case. The authors of the paper have repeatedly been in situations where interviews with various transportation companies revealed that managers had a vague idea of the effective organization of the transportation process. It was also not uncommon for their wrong actions to be discovered belatedly, when the company had already entered an unstable state due to such wrong actions [10].

In this regard, the analysis of the so-called "digital footprints" of freight transportation operators is of great practical interest. Digital footprints refer to information about events that the user performs in the transportation management information system. Modern information systems currently record all user operations in a special file (event log), which shows the date and time of the operation, the operation itself, its ID, as well as the user who performed this operation. Thus, it is actually possible to perform the so-called "backtracking" based on the records of this log, i.e. to reconstruct all the business activities that a certain user performed in the information system [11]. This approach is part of a set of methods united by the general concept of Process Mining. Currently, there are a number of software solutions, such as Celonis, Promo, Disco and others, that enable reconstruction of users' business processes in various information systems. Their functionality is extremely diverse and allows for a deep intellectual analysis of business processes of operators engaged in the fleet management information system (Fig. 1)

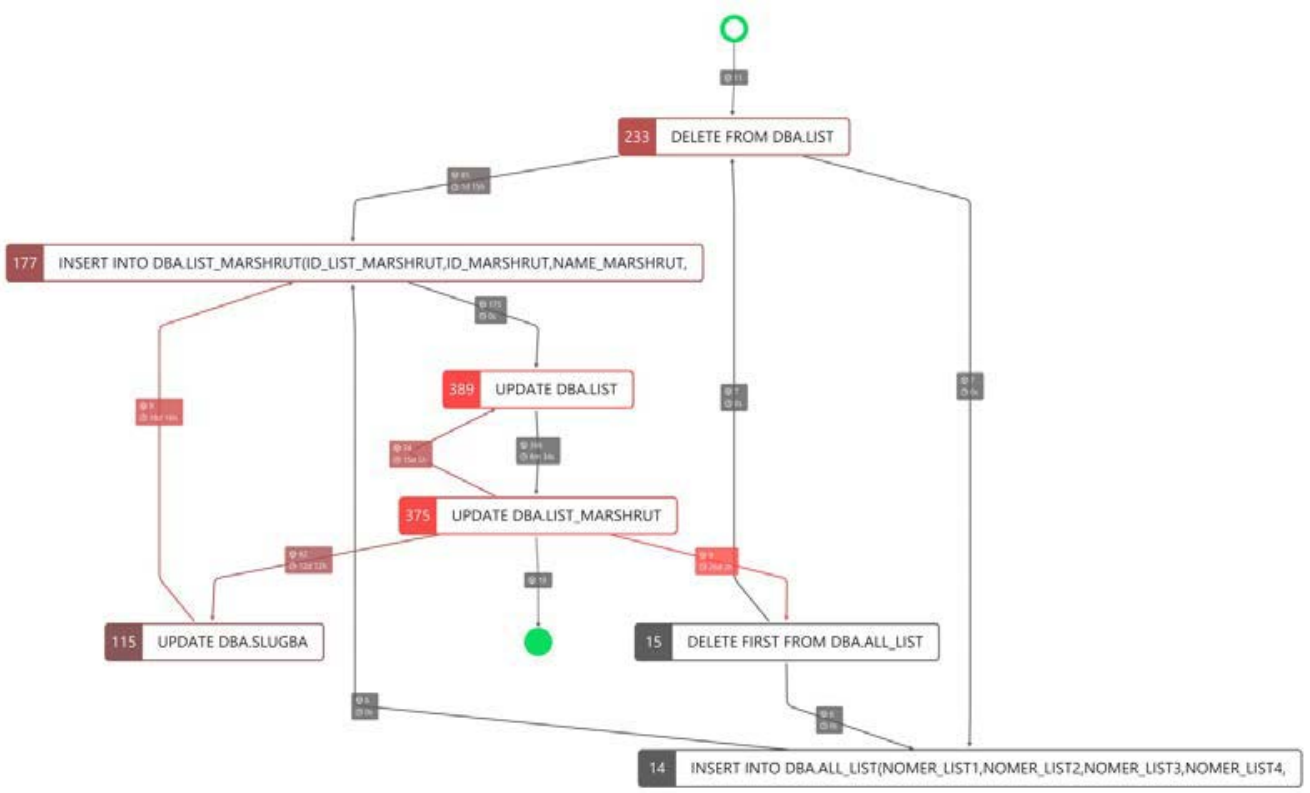

Fig. 1. Reconstructed business process of a dispatcher based on the event $\log$ in the information system [12]. 


\subsection{Use of Control Charts to Identify Business Process Variations}

However, the daily activities of a transportation company require a tool that would allow for very a quick detection of deviations from the existing business process. There are many examples where an operator commits unintentional or intentional erroneous actions in the information system. This may be caused by fatigue, lack of concentration, haste, or misunderstanding of the logic of the information system. And sometimes it can be caused by the desire to commit an unfair operation. Accordingly, managers should have an alarm signal in front of them, which is triggered when the process gets out of control.

We suggest using the Shewhart control charts for this purpose. In 1924, W. Shewhart at Bell Labs proposed using specially designed charts for statistical process control. The main idea of this method is the constant monitoring of a certain controlled parameter [13]. In the simplest case of using this method, the control limits of changes in this parameter are established based on the monitoring data: the center line (CL), the upper control limit (UCL), the lower control limit (LCL), where UCL and LCL depend on the average value of the measured indicator and the $\mathrm{R}$ span. The span is the difference between the smallest and the largest value of the measured parameter. CL is the reference value of the monitored parameter. The control limits of UCL and LCL are located at a distance of $3 \sigma$ from CL. ( $\sigma$ means the standard deviation of the controlled parameter). For the practical calculation convenience, a special tabular coefficients $\mathrm{A}_{2}$ or $\mathrm{E}_{2}$ are also used, depending on the number of values of the controlled parameter. The first one is used where groups of monitoring of the same event are available. The second one is used where the process does not involve repeated monitoring. So, CL will be defined as $\bar{x}$, and UCL and LCL will be defined as follows:

$$
\begin{gathered}
U C L=\bar{x}+E_{2} \bar{R} \\
L C L=\bar{x}-E_{2} \bar{R}
\end{gathered}
$$

W. Shewhart developed his own charts to control deviations in the physical production of any goods. However, control charts can also be used to monitor the process associated with services, for example, with freight transportation. To use Shewhart charts, it is necessary to determine the parameters that must be controlled $[14,15]$. We suggest using control charts to monitor the closing time of waybills. Based on the data from the waybill, the vehicle maintenance and transportation activities are scheduled, and transportation costs are recorded. Thus, formally, the work of a vehicle is considered completed if the fully issued waybill is timely handed over by the driver to the dispatcher immediately upon returning to the garage after performing freight transportation. If the waybills are filled in with errors or not completely, handed over later than the deadline or even lost, this causes issues with the analysis and ensuring the reliability of transportation due to the loss of information or documents.

With Process Mining tools, we found that the dispatchers do not perform the operation to close the waybills in a timely manner. Based on the analysis, it was found that in the studied company, closure is allowed within 40 hours on average after the time of return to the garage [Fig. 2]. Accordingly, the longer closing time of waybills is a deviation that affects the reliable operation of the company. We developed methodological recommendations for improving the business processes of the dispatching service of the company, which included changing the schedule of operators, reorganizing the process of drawing up waybills using RPA (Robotic Process Automation), as a result of which operators had more time for data entry from waybills into the information system in a timely and accurate manner. 


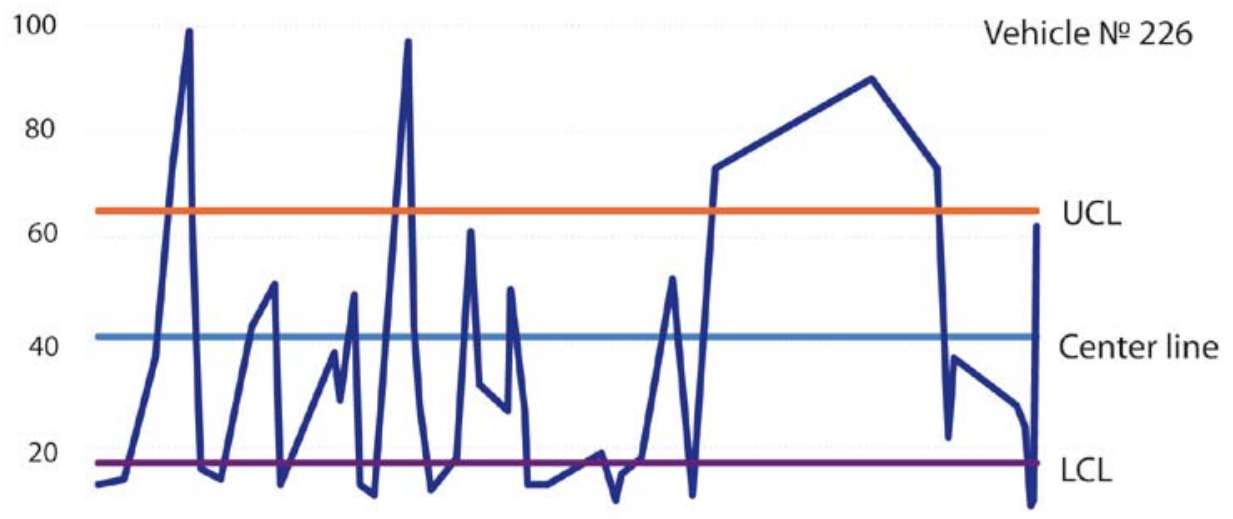

Mar. 2019 May 2019 July 2019 Sept. $2019 \quad$ Nov. 2019

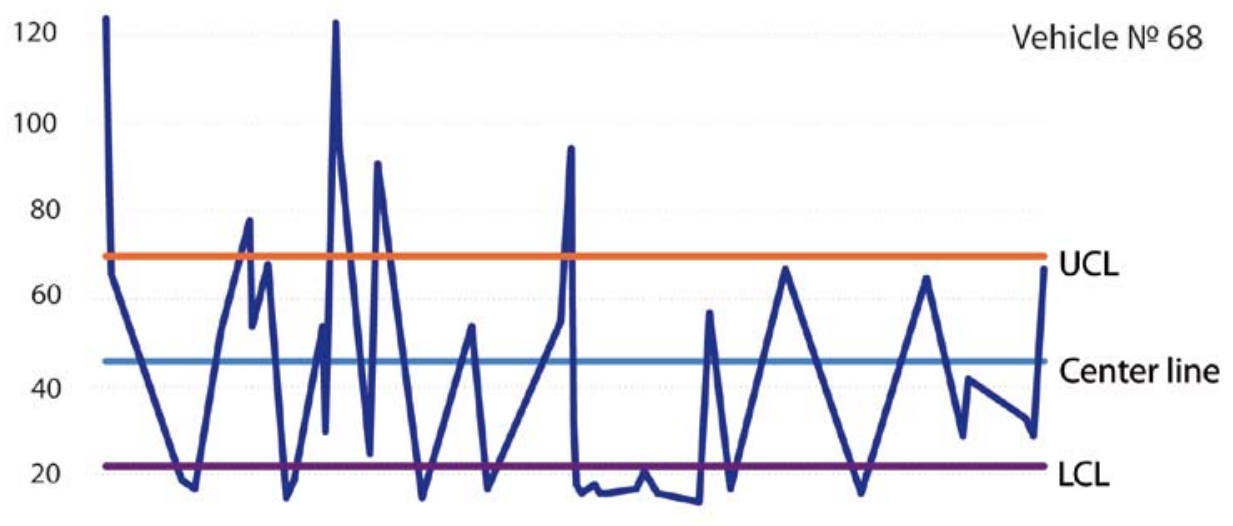

Mar. 2019 May 2019 July 2019 Sept. 2019 Nov. 2019

Vehicle № 438

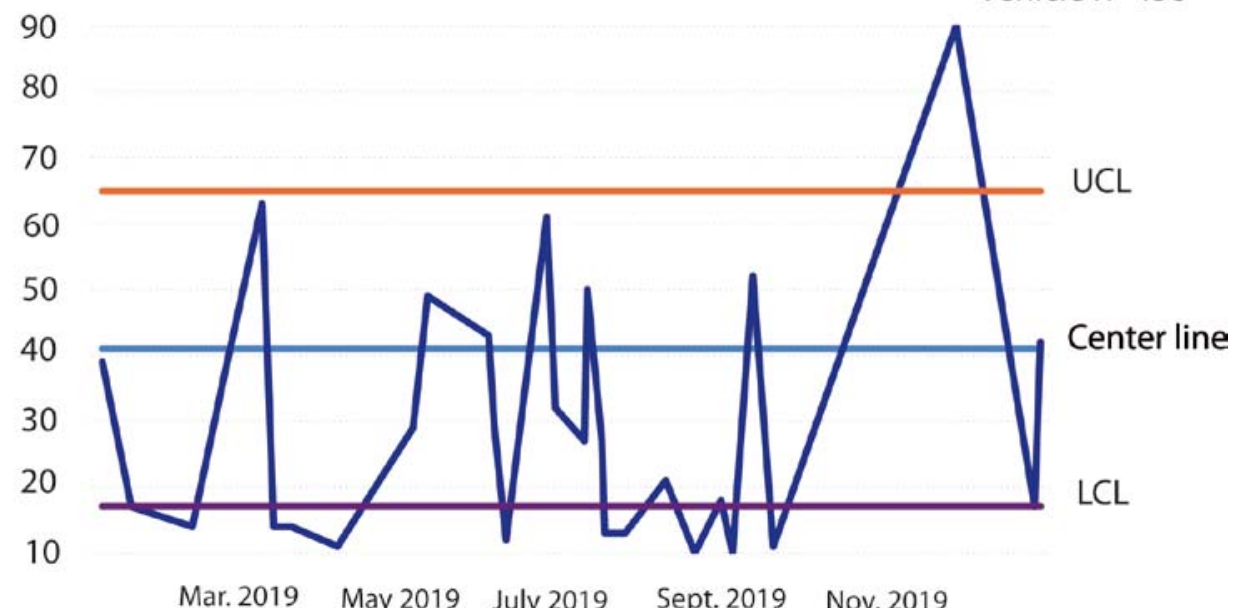

Fig. 2. Variation of the waybill closing process in the information system. 


\section{Conclusion}

Improving the reliability of road freight transportation is a complex multi-faceted task that requires consideration of various factors that affect the transportation process. One of the most important factors is the human factor, which is hard to study due to the fact that in the transportation process, the activities of management personnel as a whole are difficult to digitize and further analyze. Still, it is of practical interest to form a mechanism that provides a fast response rate to the process variation. We have proposed an approach that combines the methods of Process Mining and Shewhart control charts, which allows for identifying critical deviations in the work of the information system operators for managing the truck fleet.

\section{References}

1. K. Yang, L. Tao, J. Bai. Assessment of Flight Crew Errors Based on THERP. Procedia Engineering, 80, 9 - 58 (2014)

2. E. Hustad, M. Haddara, B. Kalvenes. ERP and organizational misfits: An ERP customization journey. Procedia Computer Science, 100, 429 - 439 (2016)

3. C.Morais, R.Moura, M.Beer, E.Patelli. Human reliability analysis-accounting for human actions and external factors through the project life cycle. In: Haugen, S. et al. (Eds.): Safety and Reliability - Safe Societies in a Changing World, 329-338 (2018)

4. J. De Haan, K.C. Terwel, S.H.S.Al-Jibouri. Design of a Human Reliability Assessment model for structural engineering. ESREL 2013: Proceedings of the 22nd European Safety and Reliability Conference "Safety, Reliability and Risk Analysis: Beyond the Horizon", Amsterdam, The Netherlands, 29 (2013)

5. B.S. Dhillon. Human Reliability and Error in Transportation Systems. Springer, 191, (2007)

6. J.A.P. Hoogervorst. Foundations of enterprise governance and enterprise engineering. Presenting the employee-centric theory of organization. Springer, 590 (2017)

7. V. Lukinskiy, V. Lukinskiy, R. Churilov. Problems of the Supply Chain Reliability Evaluetion. Transport and Telecommunication, 15, 2, 120-129 (2014)

8. A. Dorofeev, V. Kurganov, M. Gryaznov. Information Support Reliability of Transportation Systems in the Industry. Proceedings of the 7th International Conference on Information Communication and Management. ACM, 162-167 (2017)

9. R. Dubey, A. Gunasekaran, The role of truck driver on sustainable transportation and logistics. Industrial and Commercial Training, 47, 3, 127-134 (2015)

10. H.Sternberg, G. Stefansson, E. Westernberg, R. Boije af Gennäs, E. Allenström, M. Linger Nauska, Applying a lean approach to identify waste in motor carrier operations. International Journal of Productivity and Performance Management, 62, 1, 47-65 (2013)

11. C. J. Turner, A.Tiwari, R. Olaiya, Y. Xu. Process mining: from theory to practice, Business Process Management Journal, 18, 3, 493-512 (2012)

12. V. Kurganov, A. Dorofeev, M. Gryaznov, M. Yakimov. Process Mining as a Means of Improving the Reliability of Road Freight Transportations, Transportation Research Procedia, 54, 300-308 (2021)

13. D. C. Montgomery. Introduction to Statistical Quality Control, 8th Edition. Wiley, 768 (2019) 
14. Ze-C. Haung, Yun-S. Chen, Yun-K. Chung. Statistical process monitoring by using process mining. IEEE Conference Anthology (2013)

15. I. Kregel, D. Stemann, J. Koch, A. Coners. Process Mining for Six Sigma: Utilising Digital Traces. Computers \& Industrial Engineering, 153 (2021) 